\title{
Erratum: Muons in Supernovae: Implications for the Axion-Muon Coupling [Phys. Rev. Lett. 125, 051104 (2020)]
}

\author{
Robert Bollig, William DeRocco®, Peter W. Graham, and Hans-Thomas Janka
}

(Received 9 April 2021; published 6 May 2021)

DOI: 10.1103/PhysRevLett.126.189901

In this Letter, the Compton production rate was erroneously taken in the $\omega \ll m_{\mu}$ limit. Since the typical photon energy $\omega \sim 3 T \sim m_{\mu}$, Eq. (3) should use the full form of the Compton cross section, resulting in the corrected version:

$$
\Gamma^{\text {Compton }}=\frac{\alpha\left(2 g_{a \mu} m_{\mu}\right)^{2}}{4 m_{\mu}^{2}}\left[\frac{\log (1+2 x)}{2 x}-\frac{1+3 x}{\left(1+2 x^{2}\right)}\right] \frac{n_{\mu}}{e^{\omega / T}-1} F_{\mathrm{deg}}
$$

with $x=\omega / m_{\mu}$.

Applying this correction to the luminosity calculation in Eq. (6) and optical depth in Eq. (7) weakens our ultimate limits by a factor of 5. Corrected Figs. 5 and 6 are provided here. The ultimate conservative bound is changed from $g_{a \mu}<10^{-8.1} \mathrm{GeV}^{-1}$ to

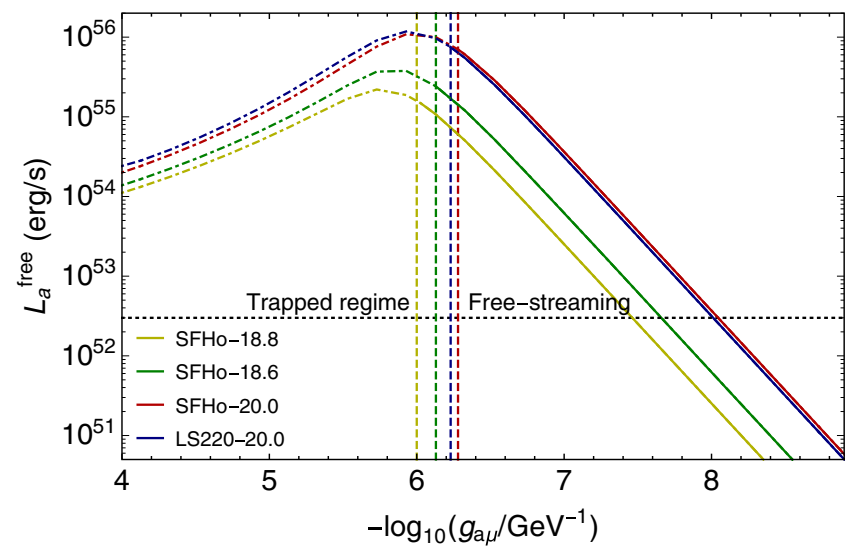

FIG. 5. The free-streaming luminosity in axions as a function of axion-muon coupling. Couplings for which the curves lie above the dotted black line and to the right of the dashed lines are excluded by the free-streaming luminosity, while couplings to the left of the vertical dashed lines are excluded by the blackbody approximation in the trapped regime. The decrease of the free-streaming luminosity toward weak couplings is due to rapidly declining axion production, whereas the decrease toward strong couplings is due to an increasing absorptive width and corresponding suppression by the $e^{-\tau(\omega, r)}$ term. Note that the free-streaming luminosity is not the total luminosity, as it neglects the contribution from blackbody surface emission when the optical depth is large. It is shown to the left of the vertical lines purely to demonstrate the expected falloff in the free-streaming (volume) emission and has hence been plotted as dotdashed lines in that region.

Published by the American Physical Society under the terms of the Creative Commons Attribution 4.0 International license. Further distribution of this work must maintain attribution to the author(s) and the published articles title, journal citation, and DOI. 


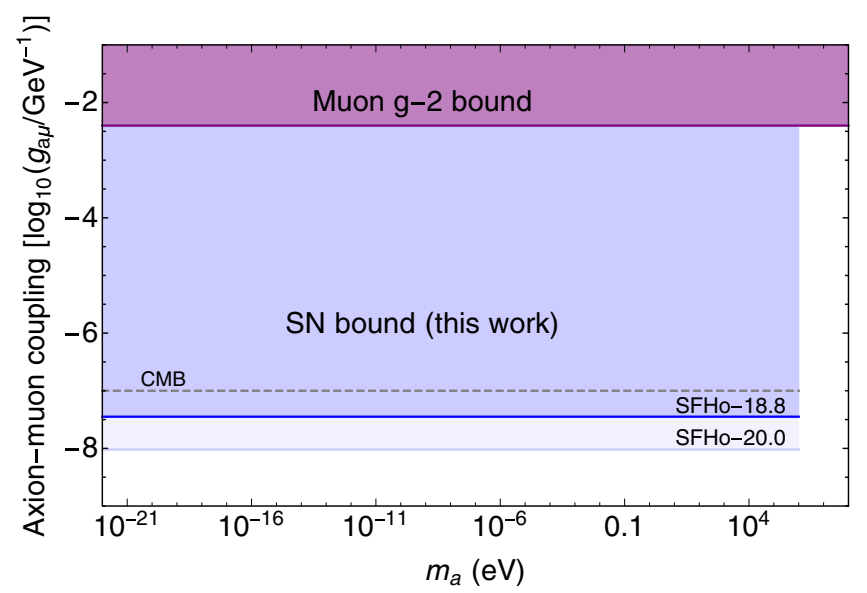

FIG. 6. Our constraints, plotted on axion parameter space. Excluded regions are colored. We show the bound that is placed with the SFHo-18.8 profile as a solid blue line, which we quote as our ultimate bound. Note that this is extremely conservative, as model SFHo18.8 is on the low edge of those compatible with SN 1987A. To illustrate the model dependence, we also show the result for SFHo-20.0 (light blue), which sits on the high edge of the allowed range. We additionally plot existing bounds from virtual axion contributions to the muon $g-2$ (purple), which are the most robust experimental constraints [20], and a rough estimate of constraints from the Cosmic Microwave Background (CMB) (dashed line, see Supplemental Material for a thorough discussion [23]).

$$
g_{a \mu}<10^{-7.4} \mathrm{GeV}^{-1} \text {. }
$$

We thank Georg Raffelt for bringing this to our attention. 